Document downloaded from:

http://hdl.handle.net/10251/36764

This paper must be cited as:

Vidal Catalá, JR.; Pla, V.; Guijarro Coloma, LA.; Martínez Bauset, J. (2013). Dynamic Spectrum Sharing in Cognitive Radio Networks Using Truthful Mechanisms and Virtual Currency. Ad Hoc Networks. 11:1858-1873. doi:10.1016/j.adhoc.2013.04.010.

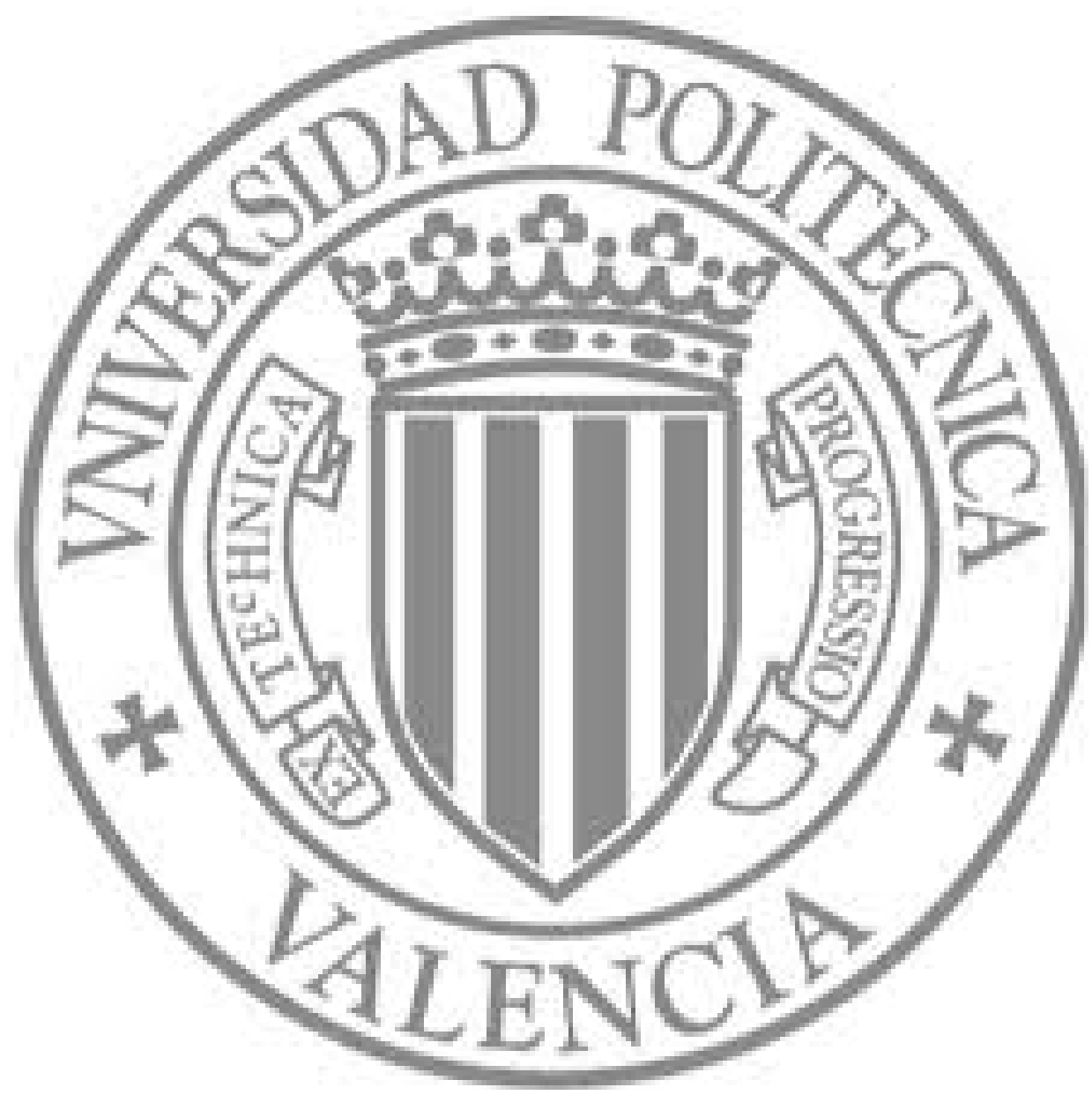

The final publication is available at

http://dx.doi.org/10.1016/j.adhoc.2013.04.010

Copyright Elsevier 


\title{
Dynamic Spectrum Sharing in Cognitive Radio Networks Using Truthful Mechanisms and Virtual Currency
}

\author{
José R. Vidal ${ }^{1, *}$, Vicent Pla, Luis Guijarro, Jorge Martinez-Bauset \\ Universitat Politècnica de València, Camí de Vera s/n, 46022 Valencia, Spain
}

\begin{abstract}
In cognitive radio networks, there are scenarios where secondary users (SUs) utilize opportunistically the spectrum originally allocated to primary users (PUs). The spectrum resources available to SUs fluctuates over time due to PUs activity, SUs mobility and competition between SUs. In order to utilize these resources efficiently spectrum sharing techniques need to be implemented. In this paper we present an approach based on game-theoretical mechanism design for dynamic spectrum sharing. Each time a channel is not been used by any PU, it is allocated to SUs by a central spectrum manager based on the valuations of the channel reported by all SUs willing to use it. When an SU detects a free channel, it estimates its capacity according to local information and sends the valuation of it to the spectrum manager. The manager calculates a conflict-free allocation by implementing a truthful mechanism. The SUs have to pay for the allocation an amount which depends on the set of valuations. The objective is not to trade with the spectrum, but to share it according to certain criteria. For this, a virtual currency is defined and therefore monetary payments are not necessary. The spectrum manager records the credit of each $\mathrm{SU}$ and redistributes the payments to them after each spectrum allocation. The mechanism restricts the chances of each SU to be granted the channel depending on its credit availability. This credit restriction provides an incentive to SUs to behave as benefit maximizers. If the mechanism is truthful, their best strategy is to communicate the true valuation of the channel to the manager, what makes possible to implement the desired spectrum sharing criteria. We propose and evaluate an implementation of this idea by using two simple mechanisms which are proved to be truthful, and that are tractable and approximately efficient. We show the flexibility of these approach by illustrating how these mechanisms can be modified to achieve different sharing objectives which are trade-offs between efficiency and fairness. We also investigate how the credit restriction and
\end{abstract}

\footnotetext{
* Corresponding author

Email addresses: jrvidal@dcom.upv.es (José R. Vidal), vpla@dcom.upv (Vicent Pla), lguijar@dcom.upv (Luis Guijarro), jmartinez@dcom.upv.es (Jorge Martinez-Bauset)

${ }^{1}$ Telephone: +34 96 3879765, Fax: +34 963877309
} 
redistribution affects the truthfulness of these mechanisms.

Keywords: wireless networks, cognitive radio, radio spectrum management, channel allocation, game theory, mechanism design

\section{Introduction}

Cognitive radio is the technology that enables dynamic spectrum access (DSA) networks to fully utilize the scarce spectrum resources [1]. In DSA networks, users who have no spectrum licenses, known as secondary users (SUs), are allowed to use the spectrum opportunistically. In this paper, we will focus on DSA networks with hierarchical and overlay access [2]. In the hierarchical access model, SUs use spectrum that is licensed to primary users (PUs). As PUs have priority in using the spectrum, when SUs coexist with PUs, they have to perform real-time wideband monitoring of the licensed spectrum to be used in order to avoid harmful interference to PUs. In overlay access, also referred to as opportunistic spectrum access, SUs only use the licensed spectrum when PUs are not transmitting. In order not to interfere with the PUs, SUs need to sense the licensed frequency band and detect the spectrum opportunities. The availability and quality of spectrum opportunities may change rapidly over time due to PUs activity, SUs mobility and competition between SUs. Therefore, dynamic spectrum allocation and sharing schemes are needed to achieve flexible spectrum access in long-run scenarios. They should be able to adapt to the spectrum dynamics, (e.g., channel variations), based on local observations.

Dynamic spectrum sharing in cognitive radios has been extensively studied from a game theoretical perspective $[3,4]$. Some works address the dynamic spectrum sharing problem using game theory with a decentralized approach $[5,6]$, in which each two SUs in a link decide how to access the available spectrum opportunities in the environment based only on the local data available to them. The decentralized approach has several advantages that make it attractive: lower complexity than the centralized approach, robustness and scalability. However, with decentralized approaches there is no guarantee that optimal solutions can be achieved. To optimize objectives, such as global efficiency and fairness, and some important parameters, such as price of anarchy and price of stability, the spectrum data for all SUs in the network should be considered. This can only be achieved by means of a centralized approach, in which the optimization problem is solved globally for the whole network. In centralized spectrum allocation, opportunistic spectrum access of SUs is coordinated by a central element serving as a spectrum manager. The spectrum manager collects operation information from each SU, and allocates the spectrum resources to achieve the objectives of efficiency and fairness.

The centralized dynamic spectrum sharing can be modelled as a non-cooperative game in which SUs only aim at maximizing their own benefit. SUs might exchange false information about their channel conditions in order to get more access opportunities to the spectrum. Therefore, cheat-proof spectrum sharing schemes should be developed to meet the objectives. Mechanism design is 
a game theoretical approach that can be applied to dynamically redistribute spectrum across several players to meet their demands. Mechanisms aim at achieving the desired equilibrium by enforcing SUs to play truthfully, so that the spectrum resources are allocated according to reliable information. This is attained by means of payments, which are collected and redistributed by a trusted entity. In spectrum auction games, a specific form of mechanism design, a spectrum manager collects bids and allocates spectrum resources to SUs and charges them according to some rules. By multiplexing spectrum supply and demand in time and space, dynamic mechanisms can improve spectrum utilization or fairness.

Most of the studies on spectrum auctions focus on the scenario where one or more PUs want to lease spectrum to SUs, and a monetary gain for PUs is involved. In [7], an auction model is proposed where a PU announces a portion of its licensed band and a unit price, and SUs bid for the desired amount of bandwidth. In [8], SUs bid for a pool of homogeneous channels announcing a demand curve, from which the auction is cleared using an approximate algorithm. A belief-assisted double auction is proposed in [9] with collusionresistant strategies based on the use of optimal reserve prices. In [10], a truthful and computationally-efficient spectrum auction is presented to support spectrum reselling from PUs to SUs with the aim of maximizing the sellers revenue by assigning spectrum to bidders who value it the most. Another solution for double auctions is presented in [11], where several PUs auction a channel each, while several SUs bid for just one of them, assuming that all the channels are homogeneous to the SUs. In [12] a resource-transaction algorithm to realize IEEE 802.22 dynamic spectrum renting is presented.

Other works propose mechanisms for power allocation in spectrum sharing. In [13] sequential second price auctions are proposed assuming complete information. In [14] an auction model is proposed where the utility of each $\mathrm{SU}$ is defined as a function of the received signal-to-noise-and-interference ratio (SINR). SUs are charged a unit price for their received SINR, so that the auction achieves the desired social utility. Mechanism design is used in [15]. There the underlay model is assumed, so SUs may transmit in the licensed spectrum when PUs are also transmitting, and their transmission power is restricted by the interference temperature limit. The objective power allocation is calculated using channel information obtained locally by SUs. A truthful mechanism with monetary transfers enforces SUs to reveal this information.

Our work differs from those cited above in three major aspects. Firstly, we address the problem of sharing spectrum opportunities between SUs in a centralized manner, controlled by a spectrum manager, but without the intervention of PUs. These opportunities appear sparse in time and space, therefore we propose mechanisms to allocate them in real time, i.e., mechanisms that have to be run every time that a spectrum opportunity appears. We describe and test two simple mechanisms (one deterministic and one randomized) for dynamic spectrum allocation which are truthful and approximately efficient, and with a very low computational complexity, so that they can be used in real-time allocations. Secondly, we investigate how these mechanisms can operate without monetary 
transactions. If monetary gain is not involved in the spectrum allocation problem, SUs payments will no longer be chosen as money, but as an alternative form of 'virtual currency'. This internal currency will be managed by the spectrum manager, which will record the credit of every SU and will distribute it to them. The operation of this scheme with virtual currency poses many challenges. The biggest one is how to get that this currency retains its value for the SUs. To achieve this, it is necessary that the mechanism itself should consider the credit kept by any SU. That is, the choice and payment functions should depend on the credit so limiting what SUs spend. Moreover, certain amount of currency units have to be injected into the system. This should be done by the manager redistributing a proper 'cash flow' to SUs. Thirdly, since the ultimate goal is not to trade with spectrum, but to share it, these mechanisms need to have the flexibility to achieve long-run objectives other than efficiency, such as fairness, maintaining their properties. We investigate how these three requirements can be satisfied and how the proposed solutions affect to the mechanisms properties. The theoretical background of this work and some preliminary experimental results were presented in [16].

The rest of the paper is organized as follows. In Section 2 we describe the model of spectrum sharing on networks with hierarchical and overlay access and with a central manager implementing a truthful mechanism. In Section 3 some background on mechanism design relevant to our problem is summarized. In Section 4 we describe two mechanisms based on the theoretical results and explain how these mechanisms can be modified to achieve fairness and to operate with virtual currency. We show and discuss experimental results in Section 5 and conclude in Section 6 .

\section{Spectrum Sharing Model}

We assume that the spectrum is divided into non-overlapping channels and that SUs are able to detect when a channel can be used without interrupting any PU. When one of these opportunities, called white spaces, appears, SUs may try to use it opportunistically. Every white space might be used by one or several SUs, with the condition of non-conflict between them. We model the interference between SUs according to the protocol interference model $[17,18]$. This model assumes that, for a given channel, $\mathrm{SU} i$ has a communication range $R_{i}$ and a larger interference range $R_{i}^{\prime}$. Let $d_{i j}$ denote the distance between $\mathrm{SU} i$ and $\mathrm{SU} j$. A transmission from $\mathrm{SU} i$ to $\mathrm{SU} j$ is successful if both of the following conditions are satisfied:

1. $d_{i j} \leq R_{i}$.

2. Any SU $k$, such that $d_{k j} \leq R_{k}^{\prime}$, is not transmitting.

A conflict graph as defined in [19] represents interference among all possible transmissions in the network. The conflict graph contains a vertex corresponding to every link in the network topology between a transmitter and a receiver. If, for a given channel, every SU has a single receiver, the conflict graph contains a vertex for each SU. We place an edge between $\mathrm{SU} i$ and $\mathrm{SU} p$ in the conflict 
graph if the corresponding links (from $\mathrm{SU} i$ to its receiver $\mathrm{SU} j$ and from $\mathrm{SU} p$ to its receiver $\mathrm{SU} q$ ) in the network can interfere each other. For the protocol model, this happens if $d_{p j} \leq R_{p}^{\prime}$ or $d_{i q} \leq R_{i}^{\prime}$.

The interference ranges depend on the transmitting power, which is imposed by the position and channel usage of nearby PUs. Additionally, the transmitting power and the distance to the receiver determines the transmission rate. Hence for a given white space, SU $i$ will be able to transmit at $B_{i}$ bps. With these assumptions, the conflicts for a given white space can be modelled by a conflict graph whose vertices correspond to the SUs which are able to use the channel. This graph, for a network of $N$ SUs, can be written in matrix form:

$$
F=\left[f_{i j}\right]_{N \times N} \text { with } \quad f_{i j} \in\{0,1\}
$$

where $f_{i j}=1$ if SUs $i$ and $j$ are in conflict, and $f_{i j}=0$ otherwise. The sharing problem in this scenario is, for each white space, to find an allocation $\vec{x}$ compatible with the conflict graph:

$$
\vec{x}=\left(x_{1} \ldots x_{N}\right) \quad \text { with } \quad x_{i} \in\{0,1\},
$$

where $x_{i}=1$ if the channel is allocated to SU $i$ and $x_{i}=0$ otherwise. The condition of non-conflict is that $x_{i} \cdot x_{j}=0$ if $f_{i j}=1$.

A single allocation $\vec{x}_{\text {eff }}$ is said to be efficient if

$$
\vec{x}_{\text {eff }}=\underset{\vec{x}}{\arg \max } \sum_{i=1}^{N} x_{i} B_{i},
$$

and we quantify the efficiency of a single allocation $\vec{x}$ using the efficiency index

$$
I_{e}=\frac{\sum_{i=1}^{N} x_{i} B_{i}}{\sum_{i=1}^{N} x_{\mathrm{eff} i} B_{i}} .
$$

For a given period, we quantify the long-term efficiency index by applying (1) to the resources accumulated by SUs during this time. If during a period $M$ consecutive allocations were made, and we denote by $\vec{x}^{j}=\left(x_{1}^{j} \ldots x_{N}^{j}\right)$ and $\left(B_{1}^{j} \ldots B_{N}^{j}\right)$ the allocations and bit-rates, respectively, at the $j$-th allocation, the most efficient aggregate allocation $\left(\vec{x}_{\text {eff }}^{1} \ldots \vec{x}_{\text {eff }}^{M}\right)$ for this period is

$$
\left(\vec{x}_{\mathrm{eff}}^{1} \ldots \vec{x}_{\mathrm{eff}}^{M}\right)=\underset{\left(\vec{x}^{1}, \ldots, \vec{x}^{M}\right)}{\arg \max } \sum_{i=1}^{N} \sum_{j=1}^{M} x_{i}^{j} B_{i}^{j} .
$$

Clearly, maximum long-term efficiency is achieved if every single allocation is efficient.

To quantify fairness of a single allocation $\vec{x}$ we employ the Jain's Fairness Index [20]

$$
I_{f}=\frac{\left(\sum_{i=1}^{N} x_{i} B_{i}\right)^{2}}{N \sum_{i=1}^{N}\left(x_{i} B_{i}\right)^{2}},
$$


whose value ranges from $1 / N$ (when a single $\mathrm{SU}$ gets all resources) to 1 (when resources are equally shared by all SUs). For a given period, we quantify the long-term fairness index by applying (2) to the resources accumulated by SUs during this time. However, the optimal long-term fairness is not obtained by maximizing fairness in every allocation, and therefore the maximizing criterion should be applied directly to aggregate allocations. The fairest aggregate allocation $\left(\vec{x}_{\text {fair }}^{1} \ldots \vec{x}_{\text {fair }}^{M}\right)$ for $M$ consecutive allocations is

$$
\left(\vec{x}_{\text {fair }}^{1} \ldots \vec{x}_{\text {fair }}^{M}\right)=\underset{\left(\vec{x}^{1}, \ldots, \vec{x}^{M}\right)}{\arg \max } \frac{\left(\sum_{i=1}^{N} \sum_{j=1}^{M} x_{i}^{j} B_{i}^{j}\right)^{2}}{N \sum_{i=1}^{N}\left(\sum_{j=1}^{M} x_{i}^{j} B_{i}^{j}\right)^{2}} .
$$

Clearly allocations cannot fulfil efficiency and fairness simultaneously. Therefore the objective will be a trade-off between long-term efficiency and long-term fairness, as defined above.

In our proposal we assume that there is a spectrum manager whose rules are abided by SUs, and there is a control channel dedicated to the communication between manager and SUs [21, 22]. Every time a white space appears, the SUs detect it and estimate their $B_{i}$ s. These estimates are made by each SU based on local information and are not available to the manager. Those SUs willing to use the channel send a valuation of the channel to the manager. The system must be designed so that SUs are encouraged to send their actual valuations of the channel $\left(\left\{B_{i}\right\}\right)$. The manager then will calculate the allocation according to the objectives, and communicate the allocation to SUs.

We assume that SUs detect and communicate which neighbours they conflict with $\left(f_{i j}\right)$, and from this the manager derives the conflict graph. We also assume that no SU can benefit from lying about the conflict graph. If SU $i$ declares nonexisting conflicts (it falsely declares $f_{i j}=1$ ), this will reduce the set of possible allocations, so it will reduce its chances to obtain the channel. On the other side, if it hides a conflict (it falsely declares $f_{i j}=0$ ), the resulting allocation may be useless for $i$ and $j$. Although there is no guarantee that the above reasoning is valid for any allocation procedure, this is a reasonable assumption in most cases, especially for those allocation procedures which favour the more efficient allocations. It seems also reasonable that the behaviour regarding the reporting of conflicts is part of the game rules which are abided by SUs. By making this assumption, the game is simplified so that the only decision that SUs make autonomously is which values of $\left\{B_{i}\right\}$ to report, and the problem becomes a type of mechanism called single parameter mechanism, as explained in Section 3. If this assumption were removed, the resulting problem would become exceedingly complex and we could not take advantage of the properties of single parameter mechanisms.

This scheme would be implementable in real time if the time necessary for an allocation was short enough in relation with the white spaces mean duration. Allocation time will be equal to the sensing time required by an SU to estimate its $B$ plus the time required by the manager to calculate the allocation and payments plus the time required to interchange messages between SUs and the manager. The sensing time is inherent to opportunistic spectrum access, and 
it would introduce a delay depending only on the state of the technology and equal for any allocation scheme. The calculation time could be negligible if a low complexity mechanism as those proposed in Section 4 is used. Regarding the messages interchanged, these are of two types: conflict-reporting messages sent by SUs to the manager to derive the conflict graph; and auction messages, those sent by SUs containing channel valuations and the response broadcasted by the manager containing the channel allocation. We assume that the conflict graph changes slower than white spaces. Therefore the graph updating could be made parallel to the allocations, and at a slower rate, so that the time required by conflict-reporting messages would not be a limiting factor. However, the auction messages propagation delay should be considered, although in local area environments it would be much shorter than the sensing time. The practical implementation of this scheme would be limited by the value of

$$
\frac{t_{s}+t_{R T T}}{\bar{t}_{w s}},
$$

where $t_{s}$ is the sensing time, $t_{R T T}$ is the round trip time in the control channel of the SU farthest to the manager, and $\bar{t}_{w s}$ is the mean duration of white spaces. This value should be considerably smaller than one for the proposed mechanism to be of practical relevance.

To calculate the allocation according to the objectives, the manager needs to know the true bit-rate estimates of all SUs. We assume that every SU estimate is the real channel capacity for this SU. Errors in the estimates could have a negative impact on the objectives of the allocation, and its magnitude would depend on the statistical distribution of the errors. This impact could become important if the estimates were biased, for example if some SUs obtain estimates which are systematically too optimistic or pessimistic. However, since here we are interested in how to encourage SUs to communicate truthfully their information to the manager, we will assume that the estimates are correct. On the other hand, SUs could benefit from lying about the channel bit-rate estimate; a higher declared value of $B_{i}$ rises the value of $\sum_{i=1}^{N} x_{i} B_{i}$ if $x_{i}=1$, so rising the manager evaluation of the efficiency of allocations including $\mathrm{SU} i$.

Example 2.1. Consider a network of six SUs with the conflict graph shown in Fig. 1. Let $\{a, b, c, d, e, f\}$ be the order in which the SUs are arranged. The conflict graph in matrix form is

$$
F=\left[\begin{array}{llllll}
0 & 0 & 1 & 0 & 0 & 0 \\
0 & 0 & 1 & 0 & 0 & 0 \\
1 & 1 & 0 & 1 & 0 & 0 \\
0 & 0 & 1 & 0 & 1 & 1 \\
0 & 0 & 0 & 1 & 0 & 0 \\
0 & 0 & 0 & 1 & 0 & 0
\end{array}\right] .
$$

Suppose that, at some white space, the bit-rate estimates of SUs are $(3,2$, $6,5,1,4)$. First, suppose and that all the SUs report the true estimates to the manager. In this case the efficient allocation will be $\vec{x}_{\text {eff }}=(0,0,1,0,1,1)$. 


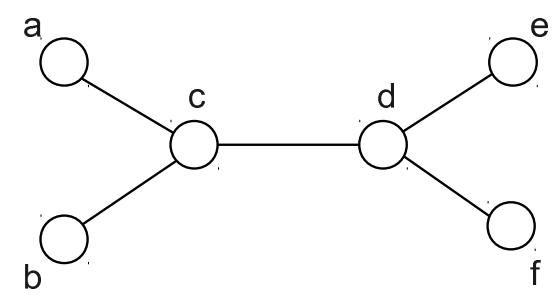

Figure 1: Conflict graph example.

But, for example, SU 4 ( $d$ in the graph) could declare an untrue value of 7 to cheat the manager. In this case, being the declared values $(3,2,6,7,1,4)$, the efficient allocation according to this information would be ${\overrightarrow{x^{\prime}}}_{\text {eff }}=(1,1,0,1$, $0,0)$, and SU 4 would benefit while the aggregated bit-rate obtained by users decreases from 11 to 10 .

We propose a solution based on mechanism design for enforcing the SUs to tell the truth to the spectrum manager driven by self-interest. The incentive to tell the truth is provided by means of payments. For each allocation, the manager will also calculate, based on the set of bit-rates reported by SUs, a price that each SU will have to pay. Thereby, the benefit obtained by an SU is the true bit-rate of the channel, if allocated to that SU, minus the price the SU pays for it. A truthful mechanism ensures that the best strategy for every SU, when behaving as a benefit maximizer, is to report the true bit-rate estimate. The price paid by SUs can be measured in monetary units, or alternatively it can be measured in a virtual currency. The first case applies when monetary transactions are desired and the main objective is to trade with spectrum. In the second case, which is the main issue of this work, the virtual currency units are distributed to SUs by the spectrum manager and they are charged after every channel allocation. Despite the fact that the currency does not have an actual monetary value, SUs will still behave as benefit maximizers because they need some credit in virtual currency to participate in the allocation game.

\section{Mechanism Design for Spectrum Sharing}

In this section we summarize the theoretical background on mechanism design underlying the mechanisms proposed in Section 4

We model the allocation procedure of each white space as a mechanism in which a spectrum manager implements the game rules and the players are the SUs. Formally, a mechanism is a game in which the players do not know the utilities of the other players, and the rules of the game are designed such that the equilibrium of the game is guaranteed to have certain desired properties. It is defined by the tuple $(\mathcal{N}, O, \Theta, \rho, u, A, M)$ :

- $\mathcal{N}$ is a set of $N$ players or, in the spectrum allocation problem, SUs.

- $O$ is the set of possible outcomes and will include the allocations. 
- $\Theta=\Theta_{1} \times \cdots \times \Theta_{N}$ is the set of possible SU types. The type of SU $i$, $\theta_{i} \in \Theta_{i}$ is known only by $\mathrm{SU} i$ and determines its utility function. Here, $\theta_{i}$ is determined by $B_{i}$.

- $\rho$ is the probability distribution of $\Theta$. Here $\rho$ depends on the position of SUs and interference restrictions.

- $u=\left(u_{1} \ldots u_{N}\right)$, where $u_{i}: O \times \Theta \mapsto \mathbb{R}$ is the utility function of SU $i$ depending on the outcome $o$ and on SU types $\Theta$. This mechanisms has the property of conditional utility independence, because the value $u_{i}$ depends only on SU $i$ type and not on other SUs type. Then, here $u_{i}: O \times \theta_{i} \mapsto \mathbb{R}$.

- $A=A_{1} \times \cdots \times A_{N}$ is the action profile, where $A_{i}$ is the set of actions available to $\mathrm{SU} i$. In this problem, allowed actions for each $\mathrm{SU} i$ are to declare a type $\hat{\theta}_{i} \in \Theta_{i}$, i.e., $A_{i}=\Theta_{i}$, what results in a so-called direct mechanism. Since SUs types are determined by the rates at which they are able to transmit in the channel, the actions available to $\mathrm{SU} i$ are the set of values of $B_{i}$ that it can declare.

- $M: A \mapsto \Pi(O)$ maps each action profile to a distribution over outcomes. If the mechanism is deterministic, then $M: A \mapsto O$.

If we want the resulting allocations to have certain properties, such as efficiency or fairness, the mechanism should implement a social function $C: u \mapsto \Pi(O)$ that maps each joint utility function to a distribution of outcomes with the desired properties. A mechanism is said to implement a social function $C$ if the game has an equilibrium in dominant strategies $a^{*}$ in which $M\left(a^{*}\right)=C(u)$. This means that the best strategy for each $\mathrm{SU} i$ is $a_{i}^{*}$. We also want the SUs to truthfully reveal their types, what will be fulfilled if the equilibrium is $a^{*}=\left(\theta_{1} \ldots \theta_{N}\right)$. In this problem, the solution can be restricted to a so-called quasilinear mechanism, where the possible outcomes can be written as

$$
O=X \times \mathbb{R}^{N}
$$

where $X$ is a finite set. In this problem, $X$ is the set of possible allocations $\vec{x}$. Hence the outcome is an allocation plus a vector of $N$ real numbers, whose $i$-th value is the price that SU $i$ has to pay for the allocation. Thus, for a given type $\theta_{i} \in \Theta_{i}$, the utility function of $\mathrm{SU} i$ is

$$
u_{i}\left(o, \theta_{i}\right)=\nu_{i}\left(\vec{x}, \theta_{i}\right)-p_{i}
$$

where $\nu_{i}\left(\vec{x}, \theta_{i}\right)$ is the valuation that allocation $\vec{x}$ has for $\mathrm{SU} i$, and $p_{i}$ is the price that SU $i$ has to pay when the allocation is $\vec{x}$. We refer to the value $\nu_{i}\left(\vec{x}, \theta_{i}\right)$ as valuation of SU $i$ for allocation $\vec{x}$, and we will write it as $\nu_{i}(\vec{x})$, considering implicit its dependence on $\theta_{i}$. Then:

$$
u_{i}\left(o, \theta_{i}\right)=\nu_{i}(\vec{x})-p_{i} .
$$


Here $\nu_{i}(\vec{x})$ can be interpreted as the maximum amount that SU $i$ would be willing to pay for allocation $\vec{x}$. Let $\nu_{i}$ denote the mapping that assigns a valuation $\nu_{i}(\vec{x})$ for each $\vec{x} \in X$. Revealing type $\theta_{i}$ is equivalent to revealing $\nu_{i}$ and the set of allowed actions for $\mathrm{SU} i$ is the set of possible values of $\nu_{i}$. Let $\hat{\nu}_{i}$ denote the declared valuation of $\mathrm{SU} i$, which might be different from $\nu_{i}$. The mechanism can be interpreted as an auction, being $\hat{\nu}=\left(\hat{\nu}_{1} \cdots \hat{\nu}_{N}\right)$ the bids, and $\vec{p}=\left(p_{1} \cdots p_{N}\right)$ the prices to pay. Let $V$ be the set of all possible vectors of valuations. Given $\hat{\nu} \in V$, the manager calculates allocation and payment from:

- A choice rule: $f: V \mapsto \Pi(X)$, or $f: V \mapsto X$ if the mechanism is deterministic.

- A payment rule: $p: V \mapsto \mathbb{R}^{N}$.

A truthful mechanism is in equilibrium in dominant strategies when $\hat{\nu}=\nu$, and no SU can benefit from declaring a false valuation. A theoretical result says that [23], for non-restricted quasilinear preferences domains, the only existing truthful mechanism is the weighted Vickrey Clarke Groves (VCG) mechanism. This is a well-known mechanism that implements efficiency. However, here we need a mechanism able to implement other criteria, such as fairness, and simple to compute. The valuation setting described before belongs to the family of single parameter domain, in which a valuation $\nu_{i}$ is defined by a single value. For single parameter domain mechanisms the former restriction does not hold since they can implement choice functions other than efficiency [24]. Formally, for each SU $i$ the set of allocations can be partitioned into a winning set $W_{i}$ and a losing set:

$$
\nu_{i}(\vec{x})= \begin{cases}v_{i} & \text { if } \vec{x} \in W_{i} \\ 0 & \text { if } \vec{x} \notin W_{i}\end{cases}
$$

Here $\vec{x} \in W_{i}$ if $x_{i}=1$, that is, SU $i$ wins if the channel is allocated to it, regardless what happens to other SUs. A deterministic single parameter domain mechanism has good properties if the choice function $f$ is monotone, that is, if an SU wins with a given valuation, it also wins with all higher valuations:

$$
\forall \hat{v}_{i}, \forall \hat{v}_{i}^{\prime}, \hat{v}_{i}^{\prime}>\hat{v}_{i}, f\left(\hat{v}_{i}, \hat{v}_{-i}\right) \in W_{i} \Rightarrow f\left(\hat{v}_{i}^{\prime}, \hat{v}_{-i}\right) \in W_{i}
$$

where $\hat{v}_{-i}$ denotes the vector of declared valuations of all SUs except SU $i$. For deterministic mechanisms with a monotone choice function, the critical value for SU $i$ is defined, given $\hat{v}_{-i}$, as the value of $\hat{v}_{i}$ below which player $i$ losses and above which player $i$ wins:

$$
c_{i}\left(\hat{v}_{-i}\right)=\sup _{f\left(v_{i}, \hat{v}_{-i}\right) \notin W_{i}} v_{i}
$$

If losing players pay 0 , the mechanism is said to be normalized. A normalized deterministic mechanism is truthful if the payment rule is [23]

$$
p_{i}\left(\hat{v}_{i}, \hat{v}_{-i}\right)= \begin{cases}c_{i}\left(\hat{v}_{-i}\right) & \text { if } \vec{x} \in W_{i} \\ 0 & \text { if } \vec{x} \notin W_{i}\end{cases}
$$


A randomized mechanism is said to be truthful on expectation if for every player, when it reveals its true valuation it maximizes its expected benefit, i.e., $\forall i \forall v_{i} \forall v_{-i} \forall \hat{v}_{i}$ :

$$
\begin{aligned}
& E\left[\nu_{i}\left(f\left(v_{i}, \hat{v}_{-i}\right)\right)-p_{i}\left(f\left(v_{i}, \hat{v}_{-i}\right)\right)\right] \geq \\
& \quad \geq E\left[\nu_{i}\left(f\left(\hat{v}_{i}, \hat{v}_{-i}\right)\right)-p_{i}\left(f\left(\hat{v}_{i}, \hat{v}_{-i}\right)\right)\right] .
\end{aligned}
$$

Let us denote by $\omega_{i}\left(\hat{v}_{i}, \hat{v}_{-i}\right)=\operatorname{Pr}\left[f\left(\hat{v}_{i}, \hat{v}_{-i}\right) \in W_{i}\right]$ the probability that SU $i$ wins when its declared valuation is $\hat{v}_{i}$ and the declared valuations for the rest of SUs is given by $\hat{v}_{-i}$. Then, the expected benefit for $\mathrm{SU} i$ is

$$
u_{i}\left(\hat{v}_{i}, \hat{v}_{-i}\right)=v_{i} \omega_{i}\left(\hat{v}_{i}, \hat{v}_{-i}\right)-p_{i}\left(f\left(\hat{v}_{i}, \hat{v}_{-i}\right)\right)
$$

If $\omega_{i}\left(\hat{v}_{i}, \hat{v}_{-i}\right)$ is monotonically non-decreasing in $\hat{v}_{i}$ and $v_{i}^{0}$ is the valuation under which $i$ cannot win, a randomized mechanism is truthful if and only if [24]

$$
p_{i}\left(\hat{v}_{i}, \hat{v}_{-i}\right)=\hat{v}_{i} \omega_{i}\left(\hat{v}_{i}, \hat{v}_{-i}\right)-\int_{v_{i}^{0}}^{\hat{v}_{i}} \omega\left(t, \hat{v}_{-i}\right) d t+h_{i}\left(\hat{v}_{-i}\right)
$$

where $h_{i}\left(\hat{v}_{-i}\right)$ is any function that does not depend on $\hat{v}_{i}$.

\section{Truthful Mechanisms with Virtual Currency}

In the spectrum sharing setting described in Section 2, a mechanism is executed to allocate a channel every time a white space appears. When SUs detect the white space, each of them estimates its valuation $v_{i}=B_{i}$. Then, each SU willing to use the channel sends a valuation of the channel $\left(\hat{v}_{i}\right)$ to the spectrum manager, using for this purpose the control channel. The manager starts an allocation procedure when it receives the first valuation and, after a fixed time interval, it closes the allocation procedure. The allocation procedure is cleared by applying the mechanism choice and paying rules to the set $\left\{\hat{v}_{i}\right\}$ of declared valuations, given a conflict graph $C$, resulting in a set of winning SUs and a vector $\vec{p}$ of payments. Finally, the manager sends a message to the winning SUs through the control channel, and charges $p_{i}$ currency units to every SU $i$.

The mechanism required belongs to the class of single parameter domain mechanisms, and it will be truthful if the choice function is monotonically nondecreasing in $\hat{v}_{i}$ for every $i$, and the payment function is the critical value for winning $S U$ s. If the mechanism is randomized, then the winning probability function must be monotonically non-decreasing in $\hat{v}_{i}$ for every $i$ and the payment rule must be given by (6). In this section we describe two simple truthful mechanisms that meet these conditions: a deterministic one and a randomized one. Then we propose some modifications to the general setting to make possible the use of virtual currency and the implementation of flexible allocation criteria. 


\subsection{Simple truthful mechanisms for spectrum sharing}

The most straightforward truthful mechanism is that which allocates the channel to SUs in sequence, starting with SUs with higher valuations, on the condition that there is no conflict with previously allocated SUs. This is a particular case of the mechanism presented in [10], when channels are allocated one at a time. In pseudo-code form, the deterministic mechanism is:

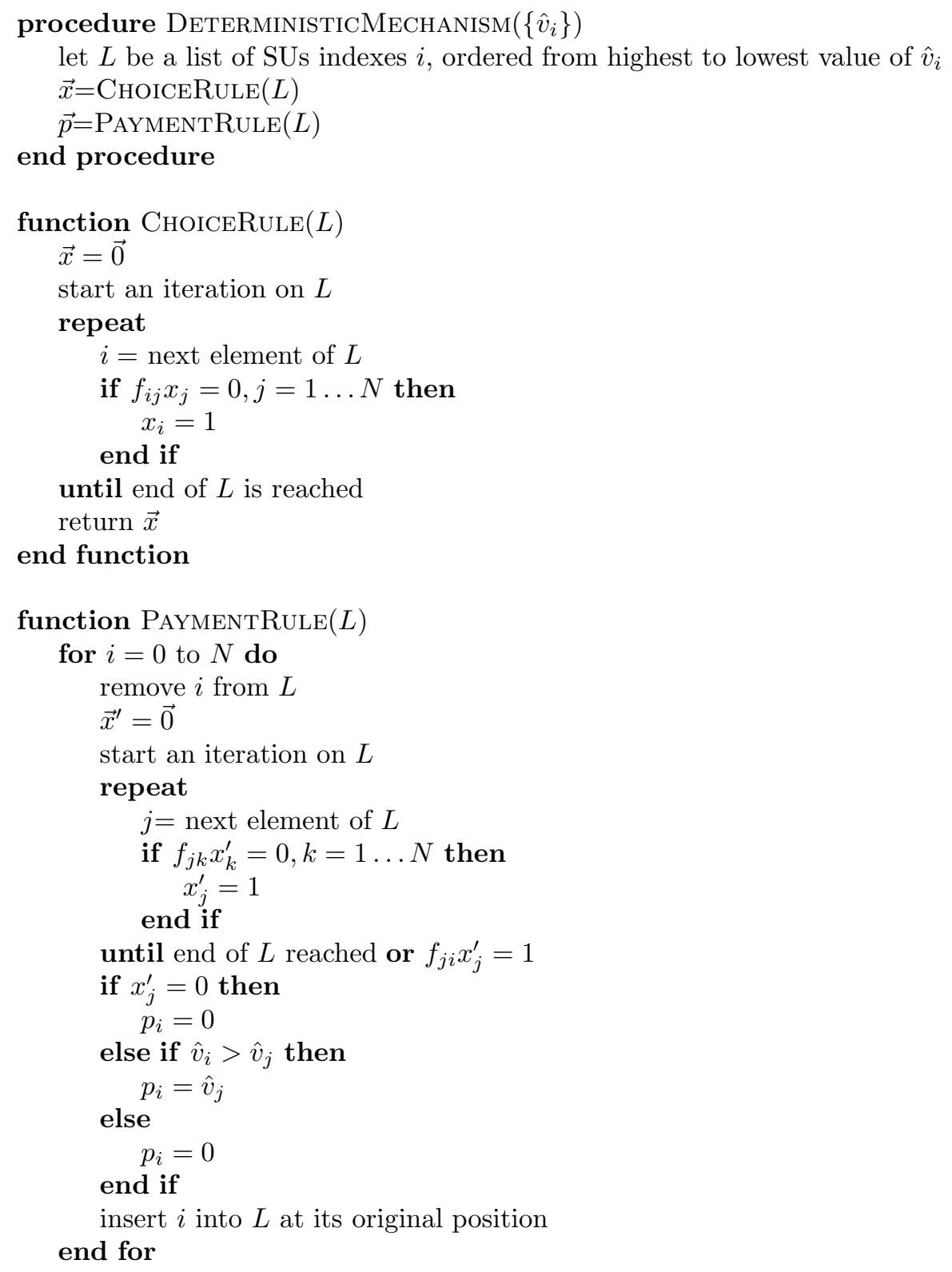




\section{end function}

The payment rule searches the critical values as defined in (5). For each $\mathrm{SU}$, its valuation is removed from the list, a virtual allocation is calculated, and its critical value is the declared valuation of the first winner, if exists, that conflicts with this SU. If an SU wins and the critical value exists, it pays this critical value. If an $\mathrm{SU}$ losses, it pays nothing. If an $\mathrm{SU}$ wins but it does not conflict with any other SU, then there is not critical value for this $\mathrm{SU}$, and it pays nothing either.

Example 4.1. Lets now apply the deterministic mechanism to the conflict graph and the bit-rate estimates of Example 2.1. First, suppose that the valuations are reported truthfully: $\overrightarrow{\hat{v}}=\vec{v}=(3,2,6,5,1,4)$. SUs indexes are ordered from highest to lowest value of $\hat{v}_{i}$ as $L=\{3,4,6,1,2,5\}$, and the choice rule results $\vec{x}=(0,0,1,0,1,1)$. The payment rule finds, for each $\mathrm{SU} i$ in $L$, the index $j$ of the first winner conflicting with $\mathrm{SU} i$ in an allocation in which $\mathrm{SU} i$ is removed:

1. SU 3: $j=4$ and $\hat{v}_{4}<\hat{v}_{3}$. The critical value is $\hat{v}_{4}$ and $p_{3}=\hat{v}_{4}=5$.

2. SU 4: $j=3$ but $\hat{v}_{3}>\hat{v}_{4}$. There is not critical value and $p_{4}=0$.

3. SU 6: The end of $L$ is reached without finding $j$. There is no critical value and $p_{6}=0$.

4. SU 1: $j=3$ but $\hat{v}_{3}>\hat{v}_{1}$. There is not critical value and $p_{1}=0$.

5. SU 2: $j=3$ but $\hat{v}_{3}>\hat{v}_{2}$. There is not critical value and $p_{2}=0$.

6. SU 5: The end of $L$ is reached without finding $j$. There is no critical value and $p_{5}=0$.

Therefore the payments are $\vec{p}=(0,0,5,0,0,0)$ and the benefits (bit-rates obtained minus amounts paid) are $(0,0,1,0,1,4)$. Suppose now that SU 4 tried to cheat the manager by reporting an untrue valuation of $\hat{v}_{4}^{\prime}=7$. By applying the mechanism to $\overrightarrow{\hat{v}}^{\prime}=(3,2,6,7,1,4)$, it would result $\vec{x}^{\prime}=(0,0,1,0,1,1)$, and SU 4 would win the channel. But now the payments would be $\vec{p}^{\prime}=(0,0,0,6,0,0)$, the benefits would be $(3,2,0,-1,0,0)$ and SU 4 would not benefit from lying.

Theorem 1. The deterministic mechanism is dominant strategy incentive compatible.

Proof. The deterministic mechanism is a normalized mechanism (payment for loosing is always 0) on a single parameter domain (the valuation is defined by a single value). Such a mechanism is dominant strategy incentive compatible if and only if the following conditions hold [23]:

(i) The choice function is monotone in every valuation as defined in (4).

(ii) Every winning user pays the critical value.

The first condition holds because the choice function depends only on the order in the list $L$. Then, if a valuation $\hat{v}_{i}$ wins, any valuation $\hat{v}^{\prime}{ }_{i}>\hat{v}_{i}$ ranks higher in $L$ and wins too. The second condition holds because the payment function 
for user $i$ finds the highest valuation $\hat{v}_{j}$ for which user $j$ conflicts with user $i$; if $\hat{v}_{i}>\hat{v}_{j}$, user $i$ wins, otherwise it losses, which is the definition of the critical value.

This mechanism can be randomized maintaining its truthfulness, with the only condition that the resulting winning probability function $\omega_{i}\left(\hat{v}_{i}, \hat{v}_{-i}\right)$ is monotonically non-decreasing in $\hat{v}_{i}$. A way of doing this, given a set of declared valuations $\left\{\hat{v}_{i}\right\}$, is by generating for each player $i$ a random value $r_{i}$ between 0 and $\hat{v}_{i}$. Then $L$ is ordered from highest to lowest value of $r_{i}$. With $L$ ordered in this way, choice and payment rules are applied. In pseudo-code form, the randomized mechanism is:

\section{procedure RANDOMIZEDMECHANISM $\left(\left\{\hat{v}_{i}\right\}\right)$}

for each $i$, calculate a random value $r_{i}$ between 0 and $\hat{v}_{i}$

let $L$ be a list of SUs indexes $i$, ordered from highest to lowest value of $r_{i}$ $\vec{x}=\mathrm{ChoiceRule}(L)$

$\vec{p}=\operatorname{RANDOMIZEDPAYMENTRULE}\left(L,\left\{r_{i}\right\}\right)$

\section{end procedure}

The choice rule for the randomized mechanism is identical to that of the deterministic one, but now it is applied to the list of SUs indexes ordered by $r_{i}$. The resulting winning probability function is monotonically non-decreasing in $\hat{v}_{i}$ because clearly the winning probability of an SU cannot decrease when its declared valuation rises. The payment rule will perform a virtual allocation for each player as in the deterministic case, but now there is no critical value, and the payments depends on the winning probability and must be calculated applying (6) to ensue the truthfulness of the mechanism. Specifically, if in the virtual allocation there is a winner $\mathrm{SU} j$ that conflicts with $\mathrm{SU} i, \hat{v}_{i}>r_{j}$, and assuming that $\left\{r_{i}\right\}$ values are generated with uniform distribution, $\mathrm{SU} i$ winning probability is

$$
\omega_{i}\left(\hat{v}_{i} \mid r_{-i}\right)=1-\frac{r_{j}}{\hat{v}_{i}}
$$

and, applying $(6)$ with $h_{i}\left(\hat{v}_{-i}\right)=0^{2}$,

$$
p_{i}=\hat{v}_{i} \omega_{i}\left(\hat{v}_{i} \mid r_{-i}\right)-\int_{r_{j}}^{\hat{v}_{i}} \omega_{i}\left(t \mid r_{-i}\right) d t=r_{j} \ln \frac{\hat{v}_{i}}{r_{j}} .
$$

However, if in the virtual allocation there is a winner SU $j$ that conflicts with $\mathrm{SU} i$ and $\hat{v}_{i}<r_{j}$, SU $i$ winning probability is

$$
\omega_{i}\left(\hat{v}_{i} \mid r_{-i}\right)=0
$$

\footnotetext{
${ }^{2}$ Here the function $\omega_{i}\left(\hat{v}_{i} \mid r_{-i}\right)$ has been used instead of $\omega_{i}\left(\hat{v}_{i}, \hat{v}_{-i}\right)$. However, the proof [24] of the condition expressed by (6) holds for both functions.
} 
and pays nothing. Finally, if in the virtual allocation there is no winner SU $j$ that conflicts with $\mathrm{SU} i, \mathrm{SU} i$ winning probability is

$$
\omega_{i}\left(\hat{v}_{i} \mid r_{-i}\right)=1
$$

and pays nothing either.

In pseudo-code form:

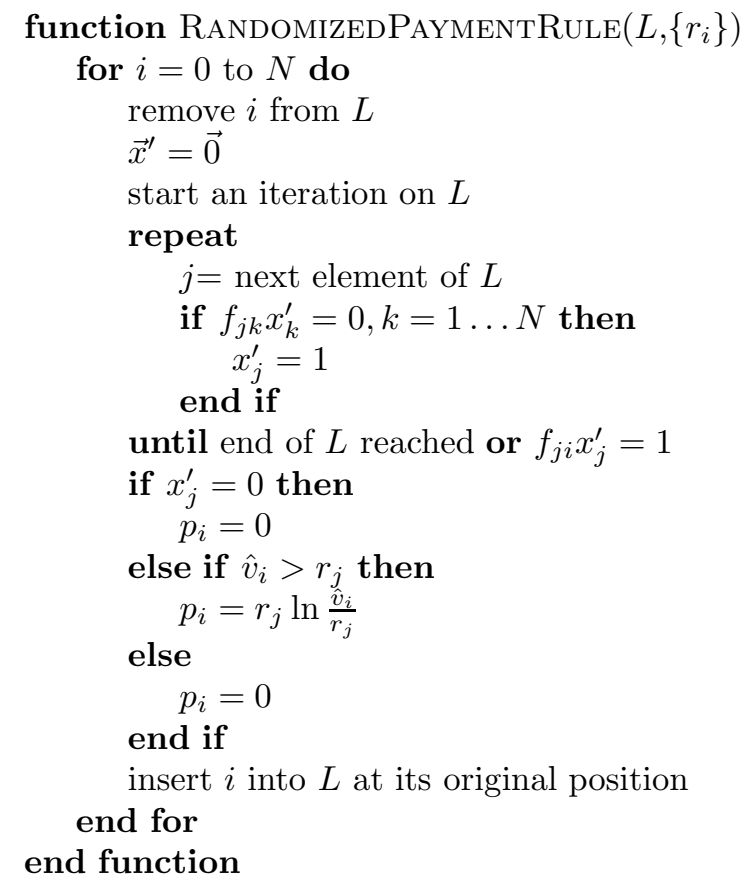

Example 4.2. Lets now apply the randomized mechanism to the conflict graph and the bit-rate estimates of Examples 2.1 and 4.1. First, suppose that the valuations are reported truthfully, $\overrightarrow{\hat{v}}=\vec{v}=(3,2,6,5,1,4)$, and that the $\left\{r_{i}\right\}$ values, obtained randomly from a uniform distribution between 0 and $\hat{v}_{i}$, are $\vec{r}=$ $(1.12,1.29,2.01,1.67,0.35,1.34)$. SUs indexes are ordered as $L=\{3,4,6,2,1,5\}$, and the choice rule results $\vec{x}=(0,0,1,0,1,1)$. The payment rule finds, for each $\mathrm{SU} i$ in $L$, the index $j$ of the first winner conflicting with $\mathrm{SU} i$ in an allocation in which $\mathrm{SU} i$ is removed:

1. SU 3: $j=4$ and $r_{4}<\hat{v}_{3}$. Then $w_{3}\left(\hat{v}_{3} \mid r_{-i}\right)=1-r_{4} / \hat{v}_{3}=0.7217$ and $p_{3}=r_{4} \ln \left(\hat{v}_{3} / r_{4}\right)=2.1358$.

2. SU 4: $j=3$ and $r_{3}<\hat{v}_{4}$. Then $w_{4}\left(\hat{v}_{4} \mid r_{-i}\right)=1-r_{3} / \hat{v}_{4}=0.5980$ and $p_{4}=r_{3} \ln \left(\hat{v}_{4} / r_{3}\right)=1.8317$.

3. SU 6: The end of $L$ is reached without finding $j$. Then $w_{6}\left(\hat{v}_{6} \mid r_{-i}\right)=1$ and $p_{6}=0$.

4. SU 2: $j=3$ and $r_{3}>\hat{v}_{2}$. Then $w_{2}\left(\hat{v}_{2} \mid r_{-i}\right)=0$ and $p_{2}=0$. 
5. SU 1: $j=3$ and $r_{3}<\hat{v}_{1}$. Then $w_{1}\left(\hat{v}_{1} \mid r_{-i}\right)=1-r_{3} / \hat{v}_{1}=0.3300$ and $p_{1}=r_{3} \ln \left(\hat{v}_{1} / r_{3}\right)=0.8050$.

6. SU 5: The end of $L$ is reached without finding $j$. Then $w_{5}\left(\hat{v}_{5} \mid r_{-i}\right)=1$ and $p_{5}=0$.

Therefore the winning probabilities are $\vec{w}=(0.33,0,0.7217,0.5980,1,1)$, the expected bit-rates (estimated bit-rates by the winning probabilities) are $(0.99,0,4.33,2.99,1,4)$, the payments are $\vec{p}=(0.8050,0,2.1358,1.8317,0,0)$ and the expected benefits (expected bit-rates obtained minus amounts paid) are $(0.1850,0,2.1942,1.1583,1,4)$. Suppose now that SU 4 tried to cheat the manager by reporting an untrue valuation of $\hat{v}_{4}^{\prime}=7$. By applying the mechanism to $\overrightarrow{\hat{v}}^{\prime}=(3,2,6,7,1,4)$, and assuming that the $\left\{r_{i}\right\}$ values obtained for $i \neq 4$ were the same as before, the resulting winning probability of SU 4 would be $w_{4}\left(\hat{v}_{4}^{\prime} \mid r_{-1}\right)=1-r_{3} / \hat{v}_{4}^{\prime}=0.7129$ and the payment would be $p_{4}=r_{3} \ln \left(\hat{v}_{4}^{\prime} / r_{3}\right)=2.5080$. Although the winning probability would increase, the expected benefit would be $5 \cdot w_{4}\left(\hat{v}_{4}^{\prime} \mid r_{-1}\right)-p_{4}=1.0565$, which is lower than before. In fact, the expected benefit of SU 4 as a function of its reported valuation is given by $5 \cdot\left(1-2.01 / \hat{v}_{4}\right)-2.01 \cdot \log \left(\hat{v}_{4} / 2.01\right)$, which has a maximum at the true valuation: $\hat{v}_{4}=v_{4}=5$.

Theorem 2. The randomized mechanism is incentive compatible in expectation.

Proof. The randomized mechanism is a normalized mechanism (payment for user $i$ with $\omega_{i}\left(\hat{v}_{i}, \hat{v}_{-i}\right)=0$ is 0 ) on a single parameter domain (the valuation is defined by a single value). Such a randomized mechanism is incentive compatible in expectation if and only if for every $i$ and every fixed $\hat{v}_{-i}$ we have that [23]:

(i) The winning probability function $\omega_{i}\left(\hat{v}_{i}, \hat{v}_{-i}\right)$ is monotonically non-decreasing in $\hat{v}_{i}$.

(ii) The payment function is $(6)$, with $h_{i}\left(\hat{v}_{-i}\right)=0$.

The first condition holds because the winning probability is given by (7), which is clearly monotonically non-decreasing in $\hat{v}_{i}$. The second condition holds because the payment function given by (8) is derived from (6) with $h_{i}\left(\hat{v}_{-i}\right)=0$.

Both mechanisms approximate efficiency by giving priority to higher valuations. Furthermore, choice and payment functions of both mechanisms are computationally tractable.

\subsection{Implementation of fairness}

The previous mechanisms can be easily modified to achieve different sharing objectives which are trade-offs between efficiency and fairness. To illustrate the flexibility of these approach, in this section we propose a heuristic modification of the mechanism in order to increase fairness.

It is easy to see that, in the original mechanisms, SUs having more competition (more neighbours conflicting with) have less chances to win and when winning, they pay a higher price. For this reason, the resulting sharing is not fair, and SUs with less competitors are favoured. We propose to compensate 
this effect by introducing a factor that favours SUs with more competition. This can be done by weighting the valuations by a function which is increasing with the number of edges in the conflict graph.

We have tested this idea by multiplying each valuation $\hat{v}_{i}$ by a weighting factor $g_{i}$ calculated as

$$
g_{i}=n_{i}^{\alpha},
$$

where $n_{i}$ is the number of edges related to vertex $i$ in the conflict graph of SU $i$ and the parameter $\alpha \geq 0$ will control the degree of fairness compensation. Simulation results are shown in Sec. 5 for several values of $\alpha$.

Clearly, the introduction of the factor $g_{i}$ does not affect the truthfulness, because the original conditions still hold. The choice functions are still monotonically non-decreasing in $\hat{v}_{i}$ and the payment function for the deterministic mechanism is the critical value. For the randomized mechanism the payment function can be easily obtained from (6), and is given by

$$
p_{i}=\hat{v}_{i} \omega_{i}\left(\hat{v}_{i} \mid r_{-i}\right)-\int_{\frac{r_{j}}{g_{i}}}^{\hat{v}_{i}} \omega_{i}\left(t \mid r_{-i}\right) d t=\frac{r_{j}}{g_{i}} \ln \frac{g_{i} \hat{v}_{i}}{r_{j}} .
$$

\subsection{Operation with virtual currency}

The main objective of spectrum sharing in this scenario is to dynamically share the available spectrum according to certain objectives of efficiency and fairness, not to trade with spectrum. For this reason, SUs should not be charged with real money, but with a virtual currency internal to the system. The virtual currency units are distributed to SUs by the spectrum manager and they are charged after every channel allocation. The manager also records the credit of each SU. SUs will still behave as benefit maximizers because they will need some credit in virtual currency to participate in the allocation game. This would also make the system simpler by avoiding monetary transactions. Furthermore, a limitation of the credit offered to SUs would help to achieve fairness.

The use of virtual currency requires the manager to implement two additional tasks:

- Credit redistribution: SUs need to receive a flow of credit along the time. At a given time, an SU could accumulate credit and it could also have a negative amount of credit, but in long-term the neat balance should be zero. If an SU accumulated positive credit, then its best strategy would be to overbid to get more chances to win. On the other side, if the system allowed an SU to accumulate negative credit with no consequences, its best strategy would be to overbid too. Therefore, in order to preserve the value of the credit unit, SUs should no accumulate credit, neither positive nor negative, when they play truthfully.

- Credit restriction: the system should limit the amount of credit that each SU spends. As in the redistribution, this is a long-term requirement. At a given time, an SU could have negative credit, but the system must 
prevent any SU from spending in long-term more than he received from the redistribution system. SUs with negative credit cannot be excluded from auctions, because they must still get their share of the redistribution and so recover credit for successive auctions. Instead, when an SU has low credit, the mechanism would grant it less chances to win.

Credit restriction and redistribution are necessary to get SUs to value the credit. Without them, the currency unit would loose its meaning because the virtual currency only has value within the system, and its value is directly related to how much channel capacity SUs can buy with it. If the value of the currency is not preserved, payments will not be an incentive any more, because the profit maximizer behaviour consists in maximizing the utility function (3), in which it is assumed that the valuations of the channel can be measured in the same units as the payments, or what is the same, that a valuation unit (bit per second) has a fixed price in currency units.

A mechanism that distributes among SUs what they pay at each allocation has a property called budget balance. Several solutions have been proposed to achieve budget balance while preserving truthfulness [25, 26, 27]. But in our problem, budget balance per allocation is not a requirement, because what we need is long-term balance between payments and redistribution, which is a much more relaxed condition. Moreover, budget balance per allocation does not guarantee individual SU balance in long-term although it guarantees that the credit of the overall system is in balance.

In order to achieve long-term balance per SU between payments and redistribution while preserving truthfulness, it is required that, for a given period, each SU may receive on expectation as much as it will spend on expectation during this period when it behaves truthfully. Because the mechanism is truthful, if an SU underbids, it will lose winning chances. On the other hand, if an SU overbids, it will accumulate negative credit and will be punished by the credit restriction system losing winning chances too. Besides, to preserve truthfulness per allocation, it is also required that the amount of credit granted to a player after an allocation be independent of its valuation declared in this allocation. By doing this, the credit granted to SU $i$ is included in the term $h_{i}\left(\hat{v}_{-i}\right)$ of the payment function in (6), and truthfulness is preserved.

A straightforward method which fulfils the above conditions is the following. For every channel allocation:

- A parallel virtual allocation is created with the same players and randomly generated valuations. Ideally, the generated valuations should have a random distribution equal to that of the real valuations.

- Every SU receives what it would pay in this virtual allocation playing truthfully.

We have evaluated experimentally this method, and simulation results shown in Sec. 5 suggest that it works when the distribution used to generate the random valuations has the same mean and variance as the distribution of the real 
valuations, although the distributions are different. To implement this method, the manager should estimate the mean and the variance of the distribution of the real valuations, based on the valuations reported in successive runs of the mechanism.

To implement credit restriction, we have added to the previous mechanisms another weighting factor $q_{i}$ which depends on credit. Valuations are weighted by $q_{i}$ and the payment functions are modified accordingly. If we consider a single channel allocation, this change preserves the truthfulness of the mechanism, as it did the weighting for fairness described in Sec. 4.2. However, this does not hold if we consider successive channel allocations, because $q_{i}$ depends on the credit of SU $i$, which in turn depends on $\hat{v}_{i}$. As a consequence, the valuation on the current allocation has an effect not only in the benefit obtained in this allocation, but also in what happens in successive allocations. For this reason, it cannot be assured that SUs could not obtain a long term benefit from lying. Because successive channel allocations are not independent, this issue should be studied as a repeated game.

We have tested the mechanisms with a weighting factor $q_{i}$ that varies linearly between 0 and 1 when the credit enters the interval $(-\beta, \beta)$, i.e.:

$$
q_{i}= \begin{cases}1 & \text { if } c_{i} \geq \beta \\ \frac{c_{i} / \beta+1}{2} ; & \text { if }-\beta<c_{i}<\beta \\ 0 & \text { if } c_{i} \leq-\beta\end{cases}
$$

where $c_{i}$ is the credit of SU $i$, and parameter $\beta$ regulates how sharply the credit limitation is applied. By doing this, SUs are forced to keep its credit not far under 0 , that is, they cannot spend much more than they receive. Simulation results shown in Sec. 5 suggest that the mechanisms with this modification still behave reasonably well, though truthfulness cannot be guaranteed in all situations.

These mechanisms described above, including credit redistribution an restriction, would work equally in dynamic scenarios in which SUs enter and leave the system. Each time an SU enters the system, it would be granted a certain amount of credit, and from the next auction, it would participate in the credit redistribution. The initial credit granted to incoming SUs should be sufficiently low as to offer them no advantage over those already in the system. This initial value could be zero or, alternatively, the average credit of all SUs currently in the system.

\section{Experimental Results and Discussion}

We have evaluated the properties of the mechanisms presented in Sec. 4 by means of discrete-event simulations, using a simulation software we developed specifically to model and simulate this problem. We have simulated a static configuration of 6 SUs with the conflict graph of Fig. 1, located into the interference range of a $\mathrm{PU}$ which conveys traffic bursts whose inter-arrival time is 


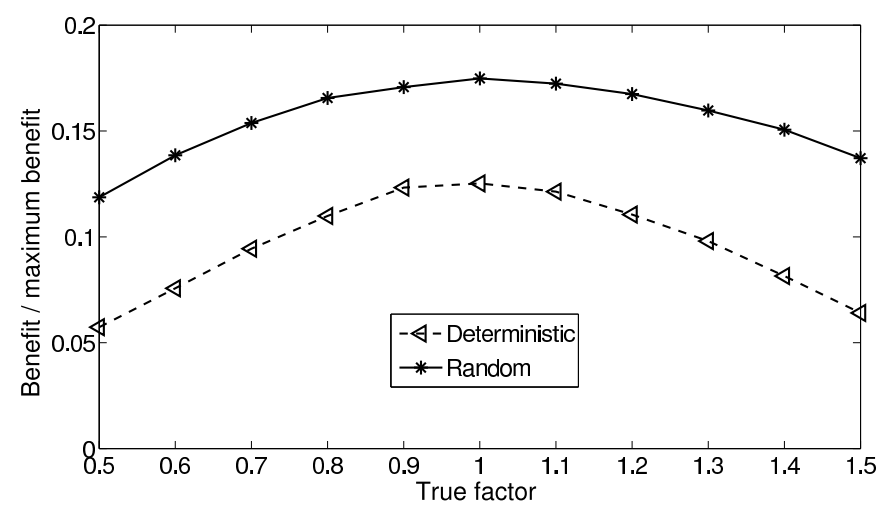

Figure 2: Benefit of SU $c$ as a function of its $t$.

exponentially distributed with mean 20 time units. The PU has a pool of 10 channels, and allocates traffic bursts randomly. The duration of these traffic bursts is exponentially distributed with mean 10 time units. We repeated these experiments for log-normally distributed traffic bursts duration and we obtained almost identical results, not shown here.

Idle periods of these channels are detected by SUs as white spaces. SUs estimates of the bit-rate of a white space are uniformly distributed between 0 and 2 bit-rate units. Every white space all SUs send their valuations of the channel to the spectrum manager, which executes the mechanism. Then the manager sends a message to the winners who occupy the channel until it is used by the PU again. The credit available for each $\mathrm{SU}$ is recorded and updated by the spectrum manager. We assume that SUs are backlogged and their objective is to send as much traffic as possible.

The plot in Fig. 2 illustrates the truthfulness of both mechanisms without credit restriction and without fairness compensation. As can be observed the results agree with the theory. Here, the benefit of SU $c$ is the sum of the bitrate of all the channels it won during the simulation run minus the total price it paid for them. The plot shows the ratio between the benefit obtained and the maximum benefit which would be obtained if the SU won all the allocations with no cost. This is plotted as a function of a true factor $t$ which measures the relation between the declared valuation and the true valuation: $\hat{v}_{c}=t v_{c}$. When $t>1$, SU $c$ is overvaluing the channel and when $t<1$ it is undervaluing it. The rest of SUs are truthful. It can be seen that $\mathrm{SU} c$ maximizes its benefit when $t=1$, i.e., when it declares the truth.

The ratio between benefit and maximum benefit shown in Fig. 2 can be interpreted as the expected fraction of available bandwidth that an SUs will obtain in long term with a given mechanism and a given configuration. We have evaluated the statistical significance of this expected value by repeating the experiment several times and calculating the confidence intervals. The plot in Fig. 3 shows these confidence intervals with a confidence level of $95 \%$ for 


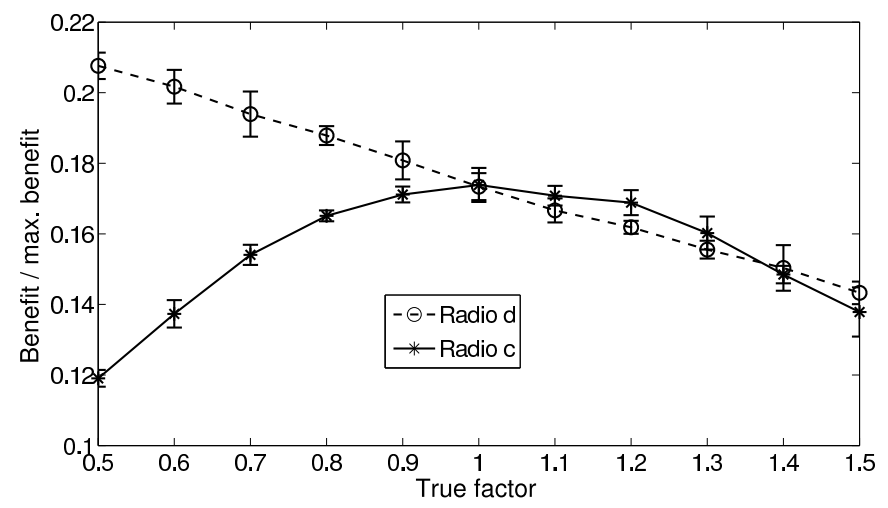

Figure 3: Confidence intervals for expected benefit with confidence level of $95 \%$ as a function of $t$ parameter on SU $c$. Deterministic mechanism.

SUs $c$ and $d$ with the deterministic mechanism. Confidence intervals for the randomized mechanism, not shown here, are very similar.

To verify that these results can be generalized to any physical configuration, we have repeated the former experiment for a large number of randomly generated topologies, with the condition that the resulting conflict graph was totally connected. Note that if an SU was disconnected from the conflict graph, it would have no competition and it would win every allocation, and then its winnings would be of no interest for our study and could hide the overall results. The plot in Fig. 4 shows the result for 5000 random connected configurations with the deterministic mechanism. Similar results have been obtained with the randomized mechanism. It can be seen that $\mathrm{SU} c$ obtains the maximum benefit when $t=1$, as expected. Besides, SUs who behave in the same way (i.e., all SUs except SU c) obtain the same result, because adverse configurations are compensated by other more favorable, and this happens for all SUs with the same probability. An interesting point is that truthful SUs benefit when SU $c$ undervalues the channels, but instead when $\mathrm{SU} c$ overvalues the channels, all SUs are harmed, including SU $c$, which suffers a more severe degradation than SUs behaving truthfully.

The capability of the mechanisms to compensate the unfairness due to disadvantage in the conflict graph, as described in Sec. 4.2, is shown in Fig. 5. This plot shows the efficiency index as a function of the fairness index for several values of parameter $\alpha$. The efficiency index has been calculated as

$$
I_{e}=\frac{\sum_{i=1}^{N} t_{i}}{\sum_{i=1}^{N} t_{i}^{\prime}},
$$

where $t_{i}$ is the amount of spectrum allocated to SU $i$ (or equivalently the traffic conveyed by SU $i$ ) with the current mechanism, and $t_{i}^{\prime}$ is the spectrum allocated to $\mathrm{SU} i$ with an efficient mechanism (as for example VCG). The fairness index 


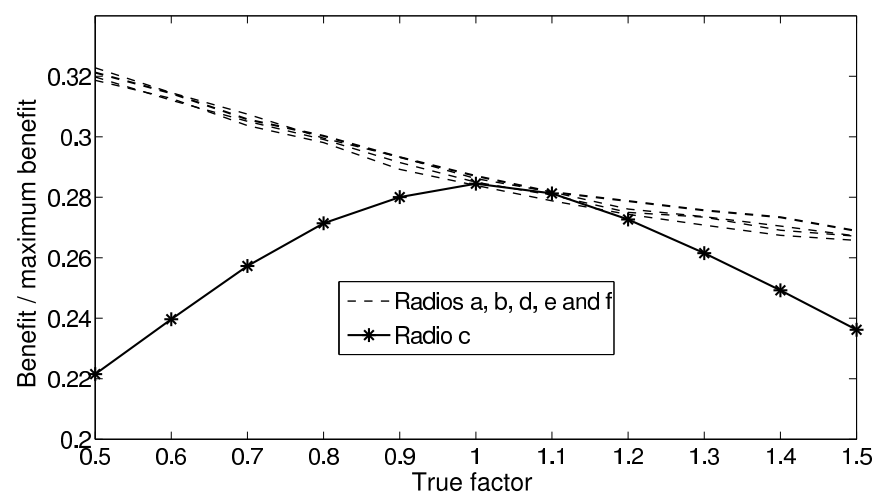

Figure 4: Average benefit over 5000 random topologies as a function of $t$ parameter on SU $c$. Deterministic mechanism.

is obtained by applying (2) to $t_{i}$ values:

$$
I_{f}=\frac{\left(\sum_{i=1}^{N} t_{i}\right)^{2}}{N \sum_{i=1}^{N}\left(t_{i}\right)^{2}} .
$$

Obviously, to improve fairness some efficiency has to be sacrificed, but these results show that an acceptable trade-off can be achieved with a high degree of fairness and without a dramatic reduction in efficiency. It is also observed that for the same degree of fairness, the deterministic mechanism yields a slightly better efficiency. Moreover, the deterministic mechanism is Pareto dominant in terms of efficiency and fairness, since for any solution reached by the randomized mechanism, there is a solution of the deterministic mechanism that is not inferior to the former in both objectives, and, additionally, there is at least one objective where it is better. However, when the random mechanism is used, fairness increases monotonically up to its maximum value as $\alpha$ varies from 0 to 1 , while when the deterministic mechanism is used, as $\alpha$ varies from 0 to 1 fairness declines after reaching the highest value. Therefore, according to these results, with the stochastic mechanism it would be simpler to adjust the trade-off between efficiency and fairness.

Credit restriction as described in Sec. 4.3 has been evaluated for several values of parameter $\beta$ and the results for SU $c$ are plotted in Figs. 6 and 7, for deterministic and randomized mechanisms respectively. Since credit is not exceeded nor accumulated, now the benefit is the obtained bit-rate, i.e., the mean traffic, and the plot shows the ratio between the benefit bit-rate obtained during the simulation run and the maximum bit-rate which would be obtained if the $\mathrm{SU}$ won all the allocations.

It can be seen that, for high values of $\beta$, the mechanism is not truthful in the long term. Specifically, with the deterministic mechanism SU $c$ can benefit from overvaluing, while whith the randomized mechanism it can benefit from undervaluing. However, both mechanisms maintain truthfulness when low values of $\beta$ are used. In fact, the best results are obtained with $\beta=0$. The 


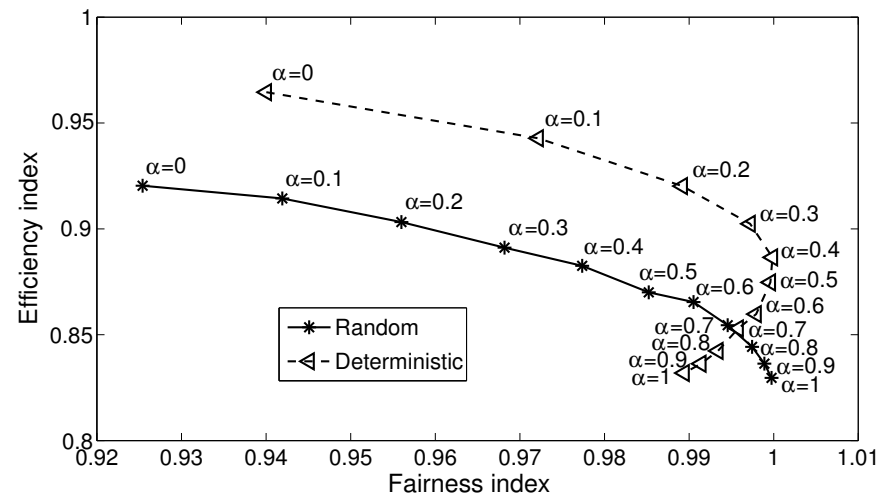

Figure 5: Efficiency index vs.fairness index as a function of parameter $\alpha$.

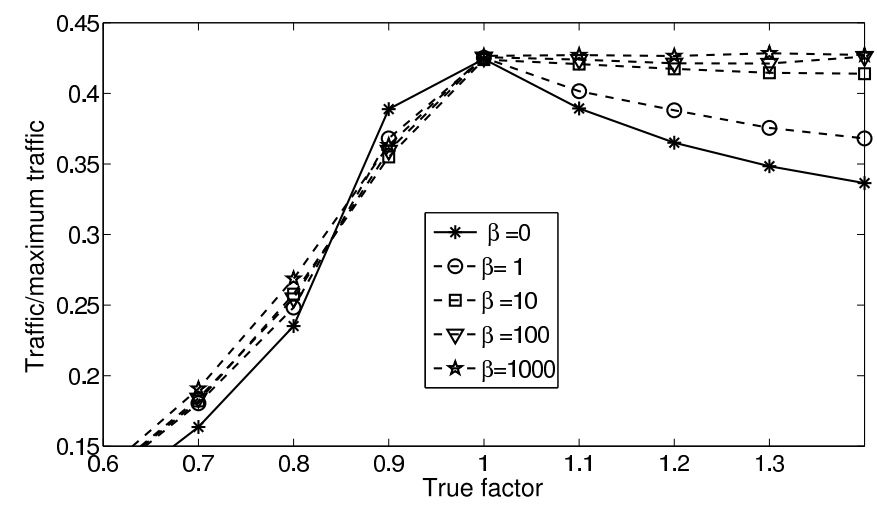

Figure 6: Traffic of SU $c$ as a function of its $t$ with credit limitation. Deterministic mechanism.

reason for this is that $\beta=0$ maintains the influence of credit in the mechanism as small as possible, that is, $q_{i}=1$ most of time while $q_{i}=0$ only when SU $i$ runs out of credit. Then, if the redistribution policy works properly, SUs will work most of time with $q_{i}=1$. When an $\mathrm{SU}$ overvalues and spends all its credit it is punished. Moreover, an SU cannot benefit from saving credit because, once it reaches enough credit to have $q_{i}=1$, having more does not increase its winning probability.

Figs. 8 and 9 show the behavior of both mechanisms with credit restriction and fairness compensation, with $\beta=0$ and $\alpha=0.4$. With these values, both mechanisms are truthful and when $\mathrm{SU} c$ behaves truthfully $(t=1)$, a high degree of fairness is obtained. Note that in this experiment, the randomized mechanism does not obtain maximum fairness because for $\alpha=0.4$ its fairness index is less than 0.98 (as shown in Fig. 5). However, for the deterministic mechanism and $\alpha=0.4$ the fairness index is close to 1 . The randomized mechanism would obtain higher fairness index with higher values of $\alpha$.

The previous results have been obtained assuming that the channel bit-rate 


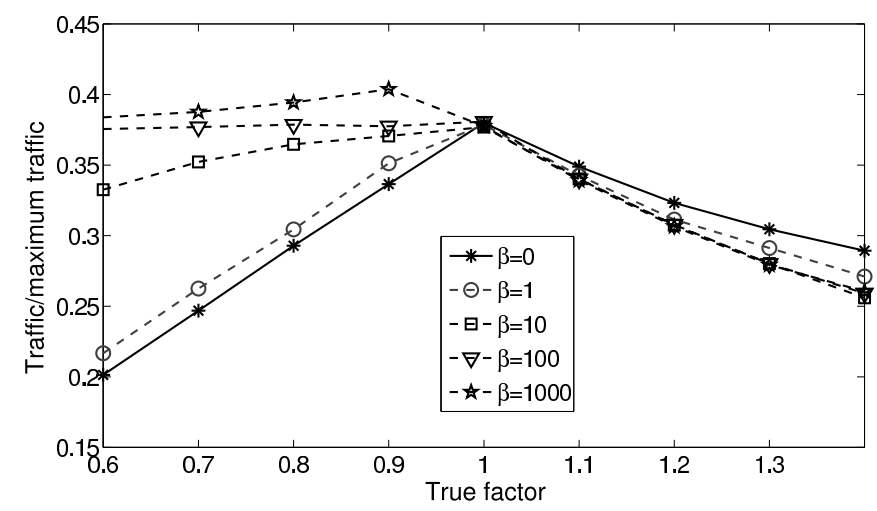

Figure 7: Traffic of SU $c$ as a function of its $t$ with credit limitation. Randomized mechanism.

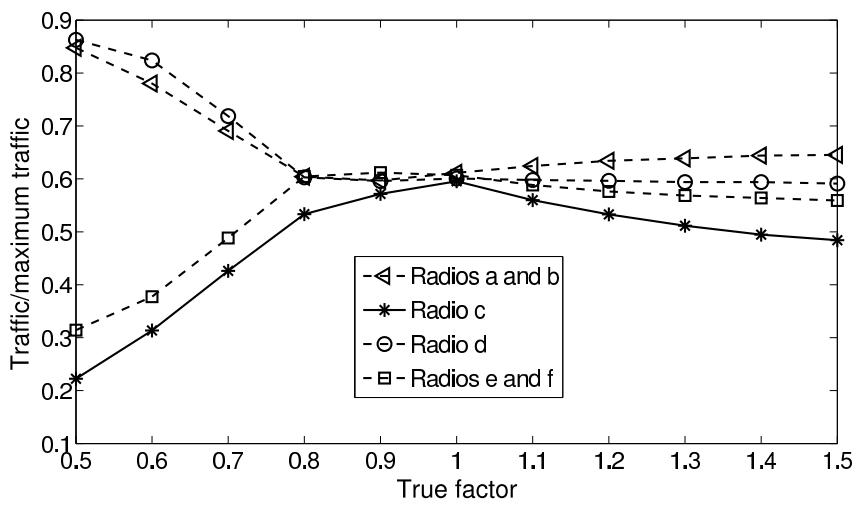

Figure 8: Traffic as a function of $t$ parameter on SU $c$. Credit limitation and fairness compensation. Deterministic mechanism.

had uniform distribution with known mean, and the random valuations used for credit redistribution were calculated with this distribution too. Figs. 10 and 11 show what happens when the channel bit-rate has a different distribution of that used to generate the random valuations. Here, we assume that channel bit-rate mean and variance are known or can be estimated, and random valuations are generated with log-normal distribution with these mean and variance. The plots show the result for SU $c$ with several channel bit-rate distributions. It can be seen that truthfulness is maintained except in case of the deterministic mechanism and uniform and Pareto channel distributions, in which a slight deviation is observed. However, the random mechanisms performs well for all the distributions tested, suggesting that randomization makes the credit redistribution less dependent on the channel bit-rate distribution, and therefore more robust. 


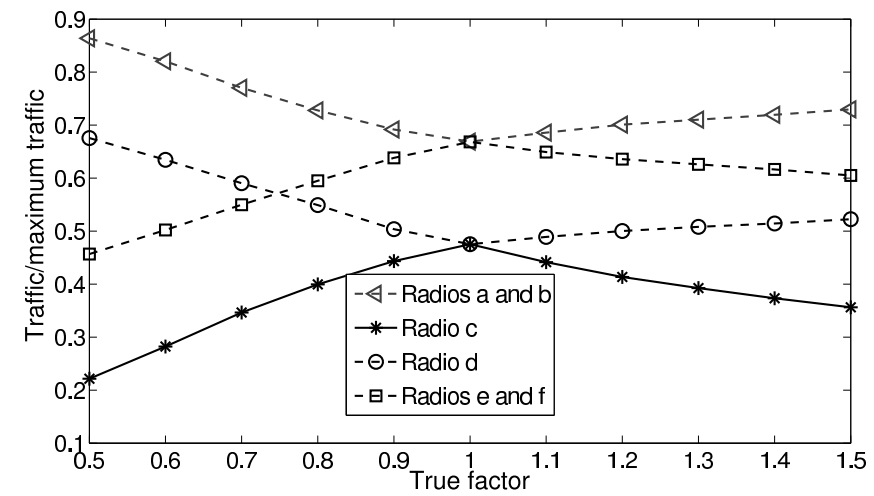

Figure 9: Traffic as a function of $t$ parameter on SU $c$. Credit limitation and fairness compensation. Randomized mechanism.

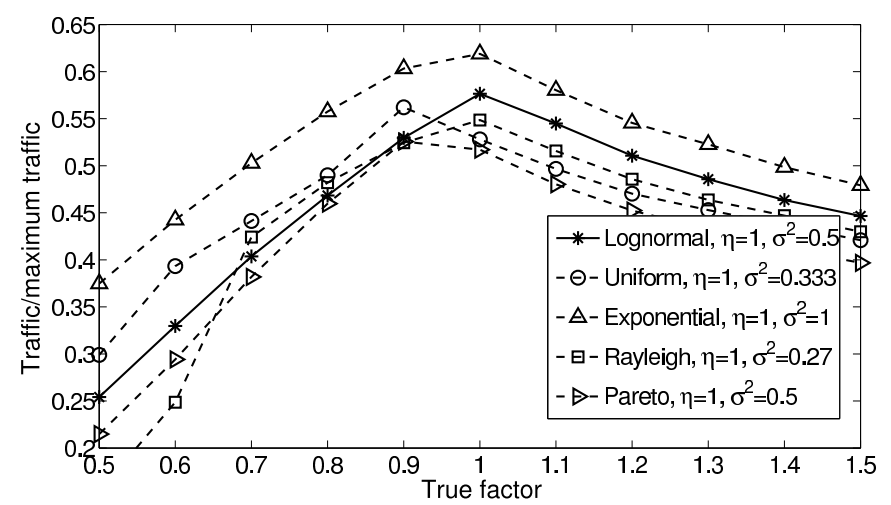

Figure 10: Traffic of SU $c$ as a function of its $t$ for several channel rate distributions. Random valuations for credit redistribution are lognormal. Deterministic mechanism.

\section{Conclusions}

We have described a dynamic spectrum sharing scheme in which SUs access opportunistically to those channels not temporarily used by PUs. Every time an available channel is detected, a centralized spectrum manager can allocate it in real-time by executing a low-complexity mechanism. Given that free channels are sensed and estimated by SUs, the mechanism should be truthful so that SUs are encouraged to communicate their true estimates to the manager. This property will allow the manager to implement the allocation according to certain objectives such as fairness or efficiency. Moreover, by using virtual currency, this scheme can operate without monetary transactions. We have tested this scheme with two simple mechanisms. Both mechanisms have been thoroughly evaluated and it is shown that their performance is independent of the physical configuration. We show how they can be modified to implement a flexible trade-off between fairness and efficiency, maintaining their properties. We 


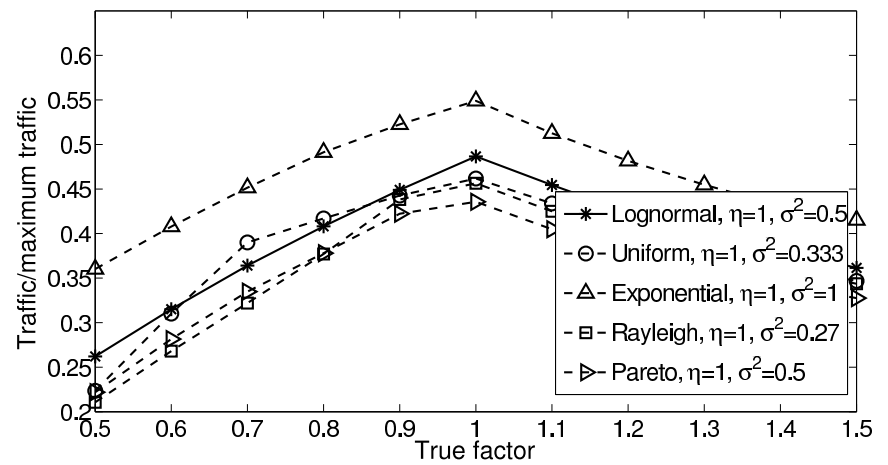

Figure 11: Traffic of SU $c$ as a function of its $t$ for several channel rate distributions. Random valuations for credit redistribution are lognormal. Randomized mechanism.

also show how they can work with a virtual currency, by controlling the credit of SUs, making the choice and payment functions dependent on the credit, and redistributing cash to SUs. When the mechanisms are dependent on credit, although they are still truthful on a single run, on repeated runs truthfulness does not hold, because successive runs become dependent. But although truthfulness cannot be guaranteed in all situations, our experimental results show that under certain conditions they still behave truthfully. One condition is that credit is redistributed to SUs according to their expected needs. This requires a previous knowledge of channel bit-rate statistics, but we found that only mean and variance are needed to maintain the mechanisms properties. We also found that the randomized mechanism has some advantages over the deterministic mechanism. One advantage is that with the randomized mechanism it is simpler to adjust the trade-off between efficiency and fairness, because in its case efficiency and fairness indices depend monotonically on the adjusting parameter. Another advantage is that with the randomized mechanism the credit redistribution policy is less dependent on the channel bit-rate distribution.

\section{Acknowledgements}

This work was supported by the Spanish government through projects TIN 2008-06739-C04-02 and TIN 2010-21378-C02-02.

\section{References}

[1] Y.-C. Liang, K.-C. Chen, G. Li, P. Mahonen, Cognitive radio networking and communications: an overview, IEEE Transactions on Vehicular Technology 60 (7) (2011) 3386 -3407.

[2] Q. Zhao, A survey of dynamic spectrum access: signal processing, networking, and regulatory policy, in: IEEE Signal Processing Magazine, 2007, pp. 79-89. 
[3] I. F. Akyildiz, W.-Y. Lee, K. R. Chowdhury, CRAHNs: Cognitive radio ad hoc networks, Ad Hoc Networks (Elsevier) 7 (5) (2009) 810 - 836.

[4] B. Wang, Y. Wu, K. R. Liu, Game theory for cognitive radio networks: An overview, Compututer Networks (Elsevier) 54 (2010) 2537-2561.

[5] I. Malanchini, M. Cesana, N. Gatti, On spectrum selection games in cognitive radio networks, in: Proceedings of the 28th IEEE conference on Global telecommunications, GLOBECOM'09, IEEE Press, 2009, pp. 284-290.

[6] F. Wang, M. Krunz, S. Cui, Price-based spectrum management in cognitive radio networks, Selected Topics in Signal Processing, IEEE Journal of 2 (1) (2008) $74-87$.

[7] X. Wang, Z. Li, P. Xu, Y. Xu, X. Gao, H.-H. Chen, Spectrum sharing in cognitive radio networks: an auction-based approach, IEEE Transactions on Systems, Man and Cybernetics 40 (2010) 587-596.

[8] S. Gandhi, C. Buragohain, L. Cao, H. Zheng, S. Suri, Towards real-time dynamic spectrum auctions, Computer Networks (Elsevier) 52 (2008) 879897.

[9] Z. Ji, K. Liu, Belief-assisted pricing for dynamic spectrum allocation in wireless networks with selfish users, in: IEEE Communications Society Conference on Sensor, Mesh and Ad Hoc Communications and Networks, Vol. 1 of SECON'06, 2006, pp. $119-127$.

[10] X. Zhou, S. Gandhi, S. Suri, H. Zheng, eBay in the sky: strategy-proof wireless spectrum auctions, in: ACM International Conference on Mobile Computing and Networking, MobiCom'08, 2008, pp. 2-13.

[11] X. Zhou, H. Zheng, TRUST: A general framework for truthful double spectrum auctions, in: IEEE International Conference on Computer Communications, INFOCOM'09, 2009, pp. 999-1007.

[12] C.-H. Ko, H.-Y. Wei, Game theoretical resource allocation for inter-BS coexistence in IEEE 802.22, IEEE Transactions on Vehicular Technology 59 (4) (2010) $1729-1744$.

[13] J. Bae, E. Beigman, R. A. Berry, M. L. Honig, R. V. Vohra, Sequential bandwidth and power auctions for distributed spectrum sharing, IEEE Journal on Selected Areas in Communications 26 (7) (2008) 1193-1203.

[14] J. Huang, R. A. Berry, M. L. Honig, Auction-based spectrum sharing, Mobile Networks and Applications (Kluwer) 11 (2006) 405-418.

[15] Y. Wu, B. Wang, K. J. R. Liu, T. C. Clancy, Repeated open spectrum sharing game with cheat-proof strategies, IEEE Transactions on Wireless Communications 8 (2009) 1922-1933. 
[16] J. R. Vidal, V. Pla, L. Guijarro, J. Martinez-Bauset, Flexible dynamic spectrum allocation in cognitive radio networks based on game-theoretical mechanism design, in: IFIP TC 6 Networking Conference, Vol. II of NETWORKING'11, 2011, pp. 164-177.

[17] P. Gupta, P. R. Kumar, The capacity of wireless networks, IEEE Transactions on Information Theory 46 (2000) 388-404.

[18] K. Jain, J. Padhye, V. Padmanabhan, L. Qiu, Impact of interference on multi-hop wireless network performance, Wireless Networks (Springer) 11 (4) (2005) 471-487.

[19] M. Marina, S. Das, A topology control approach for utilizing multiple channels in multi-radio wireless mesh networks, in: International Conference on Broadband Networks., Vol. 1, 2005, pp. 381-390.

[20] R. Jain, D.-M. Chiu, W. Hawe, A quantitative measure of fairness and discrimination for resource allocation in shared computer systems, CoRR cs.NI/9809099.

[21] C. Cormio, K. R. Chowdhury, Common control channel design for cognitive radio wireless ad hoc networks using adaptive frequency hopping, Ad Hoc Networks (Elsevier) 8 (4) (2010) $430-438$.

[22] C. Han, J. Wang, Y. Yang, S. Li, Addressing the control channel design problem: OFDM-based transform domain communication system in cognitive radio., Computer Networks (Elsevier) (2008) 795-815.

[23] N. Nisan, T. Roughgarden, E. Tardos, V. V. Vazirani (Eds.), Algorithmic Game Theory, Cambridge University Press, 2007.

[24] A. Archer, E. Tardos, Truthful mechanisms for one-parameter agents, in: IEEE symposium on Foundations of Computer Science, FOCS '01, 2001, pp. $482-491$.

[25] B. Faltings, A budget-balanced, incentive-compatible scheme for social choice, in: Agent-Mediated Electronic Commerce VI, Vol. 3435 of LNAI, Springer, 2005, pp. 30-43.

[26] M. Gonen, R. Gonen, E. Pavlov, Generalized trade reduction mechanisms, in: ACM Conference on Electronic Commerce, EC'07, 2007, pp. 20-29.

[27] D. Garg, Y. Narahari, S. Gujar, Foundations of mechanism design: A tutorial part 1-key concepts and classical results, Sadhana 33 (2008) 83130 . 

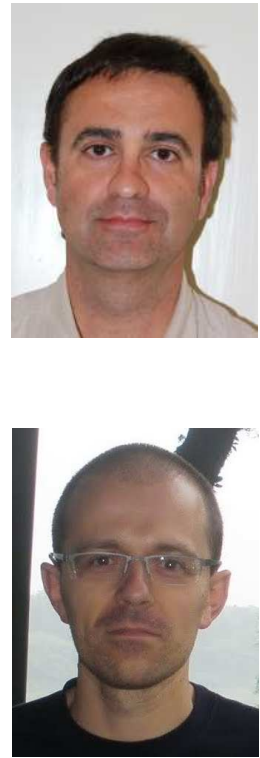

José-Ramón Vidal is currently an Associate Professor in Telematics at the Higher Technical School of Telecommunication Engineering of the Universitat Polit'ecnica de València (UPV), València, Spain. He obtained the $\mathrm{PhD}$ in Telecommunication Engineering from the UPV. His current research interest is focused on the area of application of game theory to resource allocation in cognitive radio networks. In these area he has published several papers in conference proceedings.

Vicent Pla received the M.E. and Ph.D. degrees in Telecommunication Engineering from the Universitat Politècnica de València (UPV), València, Spain. He is currently an Associate Professor with the Department of Communications at the UPV. He has published numerous papers in refereed journals and conference proceedings. His research interests include teletraffic and the modeling and performance evaluation of communication networks. During the past few years, most of his research activity has focused on resource management in wireless networks.

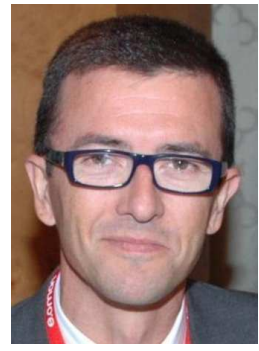

Luis Guijarro received the Ph.D. degree in Telecommunication from the Universitat Politècnica de València (UPV), Spain in 1998. He is an Associate Professor in Telecommunications Policy at the UPV. He researched in traffic management in ATM networks and in e-Government, and collaborated with the Jean Monnet Chair on Telecommunications and Information Society Policy at the European Union, based in the UPV, and published the book The Electronic Communications Policy of the European Union. His current research is focused on economic modeling of telecommunication service provision. $\mathrm{He}$ has contributed in the areas of $\mathrm{P} 2 \mathrm{P}$ interconnection, cognitive radio networks and search engine neutrality.

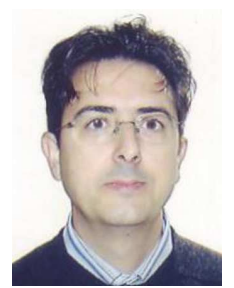

Jorge Martinez-Bauset received his Ph.D. in Telecommunication Engineering from the Universitat Politècnica de València (UPV), Spain, and currently is an associate professor at the same university. From 1987 to 1991 he was with QPSX Communications (Perth, Western Australia) participating in the design of the first IEEE 802.6 MAN. He has been with the Dep. of Communications of the UPV since 1991. He is a member of the Euro-NF Network of Excellence. He was recipient of the 1997s Alcatel Spain best Ph.D. Thesis award in access networks. His research interests are in the area of performance evaluation and traffic control for multiservice networks. In these areas he has published a number of papers in refereed journals and confer- 
ence proceedings and has participated in different research projects sponsored by European and national governments, as well as by private companies. 